\title{
Numerical simulations of nanostructured gold films
}

Repän, Taavi; Frydendahl, Christian; Novikov, Sergey M.; Beermann, Jonas; Bozhevolnyi, Sergey I.; Mortensen, N. Asger; Stenger, Nicolas; Willatzen, Morten; Lavrinenko, Andrei

\section{Published in:}

Proceedings of 17th International Conference on Numerical Simulation of Optoelectronic Devices

Publication date:

2017

Document Version

Peer reviewed version

Link back to DTU Orbit

Citation (APA):

Repän, T., Frydendahl, C., Novikov, S. M., Beermann, J., Bozhevolnyi, S. I., Mortensen, N. A., Stenger, N., Willatzen, M., \& Lavrinenko, A. (2017). Numerical simulations of nanostructured gold films. In Proceedings of 17th International Conference on Numerical Simulation of Optoelectronic Devices (pp. 5-6). IEEE.

\section{General rights}

Copyright and moral rights for the publications made accessible in the public portal are retained by the authors and/or other copyright owners and it is a condition of accessing publications that users recognise and abide by the legal requirements associated with these rights.

- Users may download and print one copy of any publication from the public portal for the purpose of private study or research.

- You may not further distribute the material or use it for any profit-making activity or commercial gain

- You may freely distribute the URL identifying the publication in the public portal 


\title{
Numerical simulations of nanostructured gold films
}

\author{
Taavi Repän ${ }^{1}$, Christian Frydendahl ${ }^{1,2}$, Sergey M. Novikov ${ }^{3}$, Jonas Beermann ${ }^{3}$, Sergey I. Bozhevolnyi ${ }^{3}$, \\ N. Asger Mortensen ${ }^{1,2}$, Nicolas Stenger ${ }^{1,2}$, Morten Willatzen ${ }^{1}$ and Andrei Lavrinenko ${ }^{1}$ \\ 1) Department of Photonics Engineering, Technical University of Denmark, Ørsteds Plads 343, DK-2800 Kongens Lyngby, Denmark \\ 2) Center for Nanostructured Graphene, Technical University of Denmark, Ørsteds Plads 343, DK-2800 Kongens Lyngby, Denmark \\ 3) Centre for Nano Optics, University of Southern Denmark, Campusvej 55, DK-5230, Odense M, Denmark
}

\begin{abstract}
We present an approach to analyse near-field effects on nanostructured gold films by finite element simulations. The studied samples are formed by fabricating gold films near the percolation threshold and then applying laser damage. Resulting samples have complicated structures, which then was captured using scanning transmission electron microscopy (STEM) and the obtained dark field images are used to set up COMSOL simulations corresponding to actual structures.
\end{abstract}

\section{INTRODUCTION}

Nanostructured gold films have complex behavior as the individual particles have various resonances due to their shape and local neighborhood [1, 2]. In recent years electron energy loss spectroscopy (EELS) has been gaining popularity as a technique to map plasmonic resonances in nanostructures [3]. Still, reasoning about such structures can be challenging, since the experimental restrictions limit available information. Numerical simulations of such structures can offer further insight into the behavior of the structure as we can choose a variety of excitations (e.g. a plane-wave or a point source) and obtain a complete picture of the fields in the structure. This gives information about polarization of the resonances and allows distinguishing bright and dark modes in the structure.

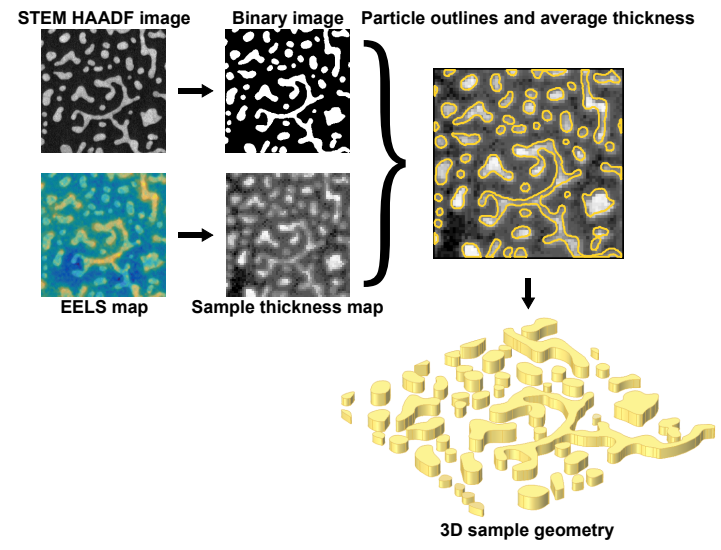

Figure 1. Outline of the simulation setup. Experimentally obtained images are used to establish the particle shapes and corresponding heights. Based on this data the $3 \mathrm{D}$ COMSOL geometry is generated.

\section{Simulation SETUP}

We study the nanostructured films with 3D full-wave simulations using the finite-element software package COMSOL Multiphysics (version 5.1). The gold particles sit on $18 \mathrm{~nm}$ thick glass substrate and we use a normally incident plane wave to excite the structure.

From experimental measurements we have 2D EELS maps and dark field STEM images (see Fig. 1). From the STEM images we estimate the shape of the gold particles that have been formed by laser damaging the nanostructured gold film. Additionally, based on the EELS measurements we estimate the height profiles of the particles. The measured EELS maps provide fine-grained resonance maps that can then be used to verify the simulation results by matching the individual resonances of the particles.

We process the STEM images to get outlines of the formed particles and generate COMSOL geometry from these outlines. Even though the initial gold film has a relatively uniform thickness, during laser damage the particles merge together and form clusters of different sizes and heights. Therefore we use measured thickness data to calculate the average height of each particle and use that to scale the particles in the simulation. For COMSOL geometry, we assume each particle to have a uniform height, and we then scale the individual particles according to the experimentally obtained height data (we average the height map values falling inside the particle contour and use that data to scale individual particles in COMSOL geometry).

We only have experimental data to estimate particle heights relative to each other, but no definite information on absolute height of the particles. For initial simulations, we start with some rough approximations using the initial deposited thickness of the gold film and change in area covered with the particles (filling fraction) after the damage. Then we compare resonances of few selected particles and scale the height to obtain a good match between simulated and measured resonance peaks.

The particles are modelled as extruded polygons with flat bottom and top surfaces (see Fig. 1). This is a simplification of the real structures, which have uneven height and less sharp walls, as they (1) are formed by partial melting and merging of several particles and (2) surface tension of molten gold forces particles to have a more round shape [4]. However, we expect that the effect of this slightly non-realistic shape is minimal and affects only the resonance position, but the general response of the particles is expected to stay the same. We verified this assumption with simulations of a simplified system - we compared a gold nanocylinder (with similar size as particles on our gold film) with an ellipsoid-like particle (having same volume) with various contact angles. These 
simulations showed that we could expect the resonance shift due to particle shape to be below $0.1 \mathrm{eV}$.

\section{RESULTS}

Firstly we used the method to study single isolated particles and verify that the behaviour seen in EELS measurements can be reproduced with our model. We focused on two particles (see Fig. 2a), one of which was a simple nanorod-like particle (particle 1), exhibiting a single resonance in the EELS spectrum. The other particle (particle 2) has a more complex shape and supports two resonances.

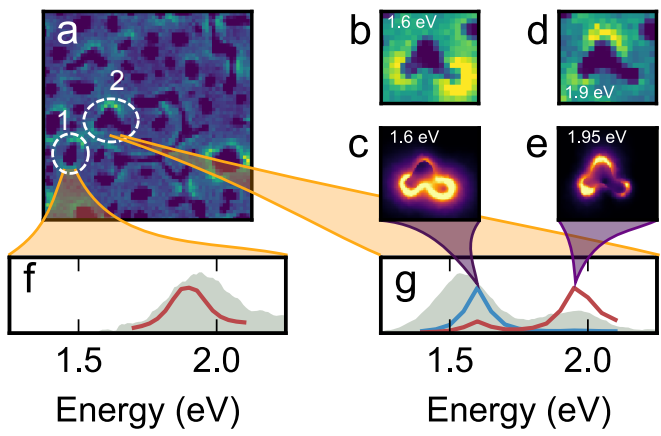

Figure 2. a) EELS map of the gold film (showing loss at $2.0 \mathrm{eV}$ ), with the two studied particles marked. b-e) Comparison of experimental EELS maps $(\mathrm{b}, \mathrm{d})$ and simulated field distributions $(\mathrm{c}, \mathrm{e})$ showing lower energy $x$-polarized mode $(\mathrm{b}, \mathrm{c})$ and slightly higher energy $y$-polarized mode $(\mathrm{d}, \mathrm{e}) . \mathrm{f}-\mathrm{g}$ ) Simulated absorption coefficients (solid lines) compared with EELS spectrums (where background has been subtracted) averaged over the particle (shown with filled areas) for particle 1 (f) and particle 2 (g). For simulated curves in (g) two polarizations have been shown ( $x$-polarization with blue and $y$-polarization with red), revealing the two different polarization modes.

Results in Fig. 2 show that the after tuning the particle heights to match the resonance positions we have a good match between data from simulations and experiment. The nanorodlike particle (particle 1) exhibits one strong resonance, seen both in simulation and experiment. The simulation also reveals that the resonance is excited by $y$-polarized light, which is expected given the orientation of the particle.

Simulations of particle 2 show that it should have two resonances at slightly different energies (and for different polarizations). The two resonances are also visible in the EELS spectrum and the corresponding EELS maps. We see a very good match between the simulation results and the experimental EELS maps (Fig. 2b-e).

In Fig. 3 we show results from another similar sample. This sample is somewhat better suited for our model, since it has (in general) smaller particles. Simulation of larger particles is more difficult as the assumption of flat surface profile is less and less realistic: larger particles are formed by merging of several smaller ones and end up with uneven surfaces, depending on the height of the initial particles.

We see that with aid of simulations we can recover information not available from experimental EELS data - as shown in Fig. 3c,f we can get information about polarization dependence of the resonances. Additionally, we could also infer information about bright and dark modes. Since we use a plane wave excitation only bright modes are present in the simulation, whereas the EELS map shows both bright and dark modes. To more conclusively differentiate between bright and dark modes that we could do additional simulations with a point source excitation so that the dark modes will also be excited. However, at this point we have not pursued this in detail.
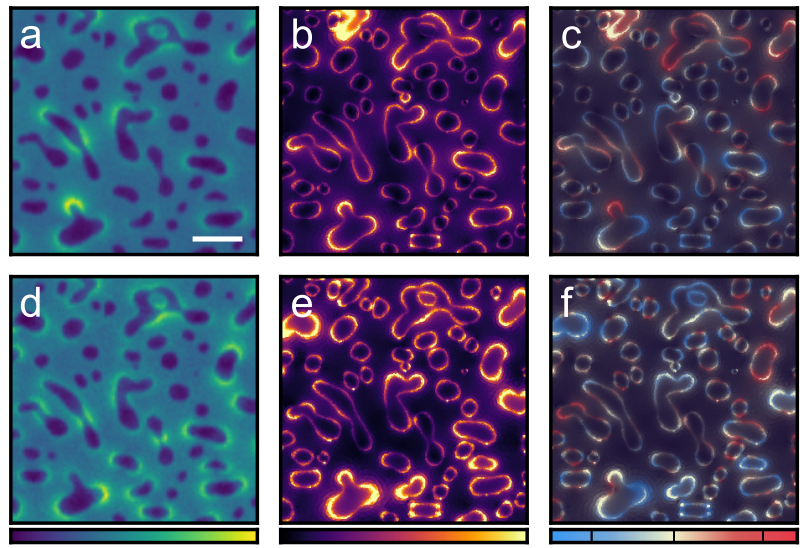

EELS intensity

Field norm (|E|) x-pol.

y-pol

Figure 3. Comparison between experimental EELS maps and simulation results. Scale bar is $100 \mathrm{~nm}$. Results for two energies are shown: $1.7 \mathrm{eV}$ (top row) and $2.0 \mathrm{eV}$ (bottom row). Corresponding simulations are done for $1.65 \mathrm{eV}$ and $1.95 \mathrm{eV}$. We show EELS maps (a,d), field norm from simulations $(\mathrm{b}, \mathrm{e})$ and also polarization maps of the resonances $(\mathrm{c}, \mathrm{f})$. In the polarization maps blue corresponds to strongly $x$-polarized response, red to $y$-polarized response and yellow to response of $x$ - and $y$-polarizations (either caused by overlap of two modes or a mode having diagonal polarization).

\section{CONCLUSION}

We show that it is feasible to generate COMSOL geometry from experimentally measured geometry and these simulations are able to capture effects visible in the experimental results. Numerical simulations also give additional insight into lightmatter interaction for nanostructured films.

\section{ACKNOWLEDGMENT}

This work has received support from Archimedes Foundation (Kristjan Jaak scholarship) and Villum Fonden (DarkSILD project).

\section{REFERENCES}

[1] S. Ducourtieux, V. Podolskiy, S. Grésillon, S. Buil, B. Berini, P. Gadenne, A. Boccara, J. Rivoal, W. Bragg, K. Banerjee et al., Physical Review B, vol. 64, no. 16, p. 165403, 2001.

[2] A. Losquin, S. Camelio, D. Rossouw, M. Besbes, F. Pailloux, D. Babonneau, G. A. Botton, J.-J. Greffet, O. Stéphan, and M. Kociak, Physical Review B, vol. 88, no. 11, p. 115427, 2013.

[3] F. G. De Abajo, Reviews of modern physics, vol. 82, no. 1, p. 209, 2010.

[4] Y. Kojima and T. Kato, Nanotechnology, vol. 19, no. 25, p. 255605, may 2008 . 